\title{
Energy dependent Schrödinger operators and complex Hamiltonian systems on Riemann surfaces
}

\author{
Mark S Alber $\dagger$, Gregory G Luther $\ddagger$ and Jerrold E Marsden $\S$ \\ $\dagger$ Department of Mathematics, University of Notre Dame, Notre Dame, IN 46556, USA \\ $\ddagger$ Department of Mathematics, University of Notre Dame, Notre Dame, IN 46556, USA and \\ the Basic Research Institute in the Mathematical Sciences, Hewlett-Packard Laboratories, Filton \\ Road, Stoke Gifford, Bristol BS12 6QZ, UK \\ $\S$ Control and Dynamical Systems 116-81, Caltech, Pasadena, CA 91125, USA
}

Received 4 April 1995, in final form 16 August 1996

Recommended by M Jimbo

\begin{abstract}
We use so-called energy-dependent Schrödinger operators to establish a link between special classes of solutions of $\mathrm{N}$-component systems of evolution equations and finite dimensional Hamiltonian systems on the moduli spaces of Riemann surfaces. We also investigate the phase-space geometry of these Hamiltonian systems and introduce deformations of the level sets associated to conserved quantities, which results in a new class of solutions with monodromy for $N$-component systems of PDEs.

After constructing a variety of mechanical systems related to the spatial flows of nonlinear evolution equations, we investigate their semiclassical limits. In particular, we obtain semiclassical asymptotics for the Bloch eigenfunctions of the energy dependent Schrödinger operators, which is of importance in investigating zero-dispersion limits of $N$-component systems of PDEs.
\end{abstract}

AMS classification scheme numbers: 58F07, 70H99, 76B15

\section{Introduction}

There are several circumstances in which semiclassical solutions and techniques are used for nonlinear evolution equations. Of particular interest is the zero-dispersion limit. Direct asymptotic expansions of the evolution equations typically yield equations of hydrodynamic type. More sophisticated techniques for integrable systems employ limiting techniques and WKB analysis in the context of the inverse scattering theory (see Lax and Levermore I, II, III (1983), Venakidis (1987) and for a survey see Lax et al (1993)). The structure of integrable equations can also be exploited using complex geometric asymptotics in this limit (see Alber 1991, Alber and Marsden 1992).

Jin et al (1994) use a WKB-type expansion for the associated scattering equations of the defocusing nonlinear Schrödinger ((d)NLS) equation and obtain equations for the conserved quantities in Riemann invariant form for the semiclassical limit $(\hbar \rightarrow 0)$. This work was extended in Bronski and McLaughlin (1994) who described the formation of caustics in a numerical context and showed that an extension of hydrodynamic type equations must be used to adequately account for the modulational instability of the focusing nonlinear Schrödinger ((f)NLS) equation. 
There have been many important developments in which the methods of complex and algebraic geometry have been used to investigate the eigenfunctions of Hill's operator in the context of integrable equations. For example, Bloch eigenfunctions of Hill's operators, which are meromorphic on the associated spectral curves, play an important role in the inverse scattering transform method for nonlinear soliton equations. For details see Ablowitz and Segur (1981), Newell (1985) and Ablowitz and Clarkson (1991).

Connections have also been established between special classes of solutions of many nonlinear equations and finite dimensional mechanical systems. Moser (1980, 1981) and Knörrer (1982) used one-dimensional Schrödinger equations with finite-gap potentials (having a finite number of bands of continuous spectrum) and reflectionless potentials (having a finite number of elements of the discrete spectrum) to demonstrate a connection between the classical Neumann problem and quasiperiodic and soliton solutions of the KdV equation. They also established a connection between the Neumann problem and the Jacobi problem of geodesics on quadrics.

For the Neumann problem, the spectral parameter appears linearly in the potential of the corresponding Schrödinger equation: $V=u-\lambda$. In contrast, Antonowicz and Fordy (1987a, b, 1988, 1989) and Antonowicz et al (1991) investigated potentials with poles in the spectral parameter for what they refer to as energy-dependent Schrödinger operators connected to certain systems of evolution equations. Specifically, they obtained multiHamiltonian structures for $N$-component integrable systems of equations related to the following isospectral eigenvalue problem:

$$
\begin{array}{ll}
L \psi=\left(\frac{\partial^{2}}{\partial x^{2}}+\frac{V}{K}\right) \psi & =0 \\
K=\sum_{j=0}^{M} k_{j} \lambda^{j} & V=\sum_{j=0}^{N} v_{j}(x, t) \lambda^{j}
\end{array}
$$

where the $k_{j}$ are constants and the $v_{j}(x, t)$ are functions of the variable $x$, the parameter $t$ and the spectral parameter $\lambda$ is complex. This includes the coupled KdV and Dym systems. The presence of a pole in the potential was shown in Alber et al (1994b, 1995) to be essential for the existence of weak billiard solutions of the nonlinear equations including the Dym equation and equations in its hierarchy. These weak billiard solutions can be obtained for all $N$-component systems.

Well known techniques exist for establishing a link between mechanical systems and their quantum mechanical counterparts. One such technique uses the WKB method to analyse asymptotic limits and thereby establish the corresponding semiclassical solutions (modes). In this paper we use this technique to establish certain semiclassical limits of the spatial flows related to integrable nonlinear evolution equations. In this way we establish a connection between special classes of solutions of nonlinear equations and mechanical systems to form a semiclassical theory for these nonlinear equations.

In what follows we make several points. We use energy-dependent Schrödinger operators of the type (1.1) and their associated spectral curves to establish a link between special classes of solutions of $N$-component systems of evolution equations and finite-dimensional Hamiltonian systems on Riemann surfaces. We apply special limiting procedures (involving the coalescence of roots of the basic polynomial of the spectral curve) to quasiperiodic solutions to obtain a system with monodromy. This provides an example of a system of PDEs with monodromy. Prior to this, such effects were related to mechanical systems and their quantum counterparts such as the classical and quantum spherical pendulum (see Cushman and Duistermaat 1988, Duistermaat 1988, Alber and Marsden 1996). 
We also describe several mechanical systems that are associated with different classes of solutions of $N$-component systems. All of these systems admit a geometric interpretation in the form of geodesic flows on $n$-dimensional pseudo-spheres in the fields of different potentials. Therefore, they can be linked to the Laplace-Beltrami operators in a way similar to the method of geometric optics. These systems are solved using algebraic geometric methods, and semiclassical approximations for eigenfunctions of Laplace-Beltrami operators having the form of complex WKB modes are studied. Using these elements we proceed to investigate the monodromy of the semiclassical approximation to the spectrum.

To investigate the zero-dispersion limit of $N$-component systems one considers the class of generalized Schrödinger equations

$$
L \psi=\left(K \frac{\partial^{2}}{\partial x^{2}}+V\right) \psi=0
$$

where

$$
K=\sum_{j=0}^{M} k_{j} \epsilon^{j} \quad V=\sum_{j=0}^{N} v_{j}(x, t) \lambda^{j} .
$$

These are similar to (1.1) and (1.2) except that the spectral parameters in the expansions of $K$ and $V$ now have a different form. The parameter $\epsilon$ will be our small parameter in this study. In this paper we investigate the asymptotic limit $\epsilon \rightarrow 0$ of the associated Bloch eigenfunctions. Using this idea, we link Jacobi geodesic flows on quadrics and the WKB approximation of the eigenfunctions of the stationary one-dimensional Schrödinger equation.

Finally, we introduce time $t$ into the system as a parameter and describe the dependence of the WKB modes on this parameter.

\section{Hamiltonian systems on Riemann surfaces and solutions of nonlinear equations}

First we recall a link between quasiperiodic and soliton solutions of integrable nonlinear equations and Hamiltonian systems on Riemann surfaces (see, for example, Alber et al 1994, 1995) established using algebraic geometric methods (see, amongst others, Ercolani and McKean 1990). Both the inverse scattering transform method for integrable systems (see Ablowitz and Segur 1981) and the method of generating equations use a link between ends of the gaps of the continuous spectrum and elements of the discrete spectrum of the Schrődinger operators and first integrals for integrable systems. Potentials in the Schrődinger operator which yield finite-gap structure of the spectrum and discrete spectrum are called finite-gap potentials and reflectionless potentials respectively.

The quasiperiodic solutions of many integrable nonlinear equations can be described in terms of finite-dimensional Hamiltonian systems on $C^{2 n}$. A complete set of first integrals for such equations can be obtained, for example, by the method of generating equations, as is summarized in Alber et al (1994, 1995). The method of generating equations introduces a finite dimensional complex phase space $C^{2 n}$ and two commuting Hamiltonian flows. The first Hamiltonian flow gives the spatial evolution, and the other gives the temporal evolution of special classes of solutions of the original partial differential equation. The level sets of the first integrals are Riemann surfaces having branch points parameterized by the choice of values of the first integrals.

Consider the finite-gap spectrum of the operator (1.1). (For details about the time evolution of the eigenfunctions of generalized Schrödinger equations of this type and multiHamiltonian structures associated with them, see Antonowicz and Fordy $(1988,1989)$.) In 
some cases, such as the $\mathrm{KdV}$ equation, $\lambda$ appears as an eigenvalue and one ultimately equates the potential with a quasiperiodic solution of the nonlinear equation itself. In other cases such as the NLS equation, a connection between the potential and solutions of the equations is more complicated but can be established using the method of generating equations.

To carry out the procedure, one begins by looking for a solution $A$ of the Lax system

$$
\begin{aligned}
& L \psi=0 \\
& \left(\frac{\partial L}{\partial t}+[L, A]\right) \psi=0
\end{aligned}
$$

of the form

$$
A=B \frac{\partial}{\partial x}-\frac{1}{2} \frac{\partial B}{\partial x}
$$

where $L$ is defined in (1.1). Substituting the given form of $A$ into the Lax system, one obtains

$$
\frac{\partial V}{\partial t}=-\frac{B^{\prime \prime \prime}}{2}+2 B^{\prime} \frac{V}{K}+B \frac{V^{\prime}}{K}
$$

where the prime denotes $\partial / \partial x$. In what follows we will also sometimes use a dot to denote $\partial / \partial t$, so $\dot{V}=\partial V / \partial t$.

Equation (2.3) is called the generating equation. Expanding $B$ and $V$ in the parameter $\lambda$ and equating like powers produces different hierarchies of integrable systems. In many cases these expansions are polynomial in $\lambda$ with coefficients depending on functions $v_{j}$ and a finite number of their space derivatives as well as one time derivative. Note that a fixed potential $V$ produces a recurrence relation for one hierarchy of evolution equations. For instance, if we take $B$ to be polynomial of degree $n$, defining $B=B_{n}=\sum_{j=0}^{n} b_{j} \lambda^{n-j}$, we obtain the $n$th system of evolution equations with dependent variables $v_{j}$ (see (1.2)).

Stationary solutions of any member of the hierarchy such that $\partial V / \partial t=0$ depend on $B$ and $V$, but the evolution variable, $t$, becomes a parameter. Setting $\partial V / \partial t=0$ in (2.3), multiplying by $B K$ and integrating once produces the stationary generating equation,

$$
K\left(-B^{\prime \prime} B+\frac{B^{\prime 2}}{2}\right)+2 B^{2} V=C(\lambda)
$$

where $C(\lambda)$ is a polynomial of $\lambda$ of the order of $(2 n+N)$. A similar equation was first obtained in connection with the KdV equation in Gelfand and Dikey (1975) by investigating asymptotics of resolvents of the Sturm-Liouville equations.

The evolution of $B$ is obtained using a dynamic generating equation. At each instant, that is for each value of the parameter $t, B$ is a solution of the the stationary generating equation (2.4). Differentiating (2.4) with respect to $t$ and requiring consistency with (2.3) yields,

$$
\dot{B}_{n}=B_{n}^{\prime} B_{l}-B_{l}^{\prime} B_{n} \quad \text { i.e. } \frac{\partial}{\partial t}\left(\frac{1}{B_{n}}\right)=\frac{\partial}{\partial x}\left(\frac{B_{l}}{B_{n}}\right)
$$

where $B_{l}=\sum_{j=0}^{l} b_{j} \lambda^{n-j}$ is a solution of the dynamical generating equation (2.3). This determines the time evolution of the functions $B$. A proof is given, for example, in Alber and Alber (1987) and Alber et al (1994). Here the $n$ is calculated using the number of roots of the spectral polynomial. These may be endpoints of gaps or isolated poles corresponding to solitons. Thus, $n$ is the dimension of the solution space and $l$ labels the equation in the hierarchy.

To show how the generating equations yield the quasiperiodic $n$-gap solutions of evolution equations, one introduces the roots, $\mu$, of $B=B_{n}=\prod_{j=1}^{n}\left(\lambda-\mu_{j}(x, t)\right)$. 
Substituting $\lambda=\mu_{j}, j=1, \ldots, n$ one by one into (2.4) one obtains a system of ordinary differential equations for the spatial flow of the $\mu$-variables:

$$
\mu_{j}^{\prime}=\frac{1}{\prod_{r \neq j}^{n}\left(\mu_{j}-\mu_{r}\right)} \sqrt{\frac{C\left(\mu_{j}\right)}{K\left(\mu_{j}\right)}} \quad j=1, \ldots, n .
$$

The motion in $t$ is produced by substituting $\lambda=\mu_{j}$ with $B$ this time in (2.5) so that,

$$
\dot{\mu}_{j}=\frac{B_{l}\left(\mu_{j}\right)}{\prod_{r \neq j}^{n}\left(\mu_{j}-\mu_{r}\right)} \sqrt{\frac{C\left(\mu_{j}\right)}{K\left(\mu_{j}\right)}} \quad j=1, \ldots, n .
$$

Solutions of this system of equations for $\mu_{j}(x, t)$ can be related to the $v_{j}(x, t)$ to obtain solutions of the original hierarchies of evolution equations generated by (2.3). Basic facts about these systems are therefore of great interest. The systems (2.6) and (2.7) have a Hamiltonian structure with

$$
H=\sum_{j=1}^{n} \frac{D\left(\mu_{j}\right)\left(P_{j}^{2}-C\left(\mu_{j}\right) / K\left(\mu_{j}\right)\right)}{\prod_{r \neq j}^{n}\left(\mu_{j}-\mu_{r}\right)} \quad j=1, \ldots, n
$$

where $D\left(\mu_{j}\right)=1$ and $D\left(\mu_{j}\right)=B_{l}\left(\mu_{j}\right)$ in the stationary and dynamical cases, respectively. The two Hamiltonian flows have the same set of first integrals, whose zero level sets are,

$$
P_{j}^{2}=\frac{C\left(\mu_{j}\right)}{K\left(\mu_{j}\right)} \quad j=1, \ldots, n .
$$

One can think of $C^{2 n}$ as being the cotangent bundle of $C^{n}$, with configuration variables $\mu_{1}, \ldots, \mu_{n}$ and with canonically conjugate momenta $P_{1}, \ldots, P_{n}$. The two commuting Hamiltonians on $\boldsymbol{C}^{2 n}$ both have the form

$$
H=\frac{1}{2} g^{j j} P_{j}^{2}+V\left(\mu_{1}, \ldots \mu_{n}\right)
$$

where $g^{j j}$ is a Riemannian metric on $C^{n}$. The two Hamiltonians are distinguished by different choices of the metric but they have the same set of first integrals (2.9). Thus, two commuting flows are obtained on the symmetric product of $n$ copies of the Riemann surface

$$
\mathcal{R}: P^{2}=\frac{C(\mu)}{K(\mu)}
$$

defined by the first integrals. It is called the spectral curve associated with the completely integrable problem. Branch points of the Riemann surface are given by the roots of $C(\lambda)$ and $K(\lambda)$. (For details about completely integrable systems on complex tori see Ercolani and McKean (1990).) These Riemann surfaces can be regarded as complex Lagrangian submanifolds. This is called the $\mu$-representation of the problem. Sometimes the $\mu$ variables are also called Dirichlet eigenvalues or an auxiliary spectrum. The $\mu$ variables move along cycles on the corresponding Riemann surface in accordance with the equations (2.6) and (2.7), which define their motion over the basic cuts on the Riemann surface whose endpoints are the endpoints of the gaps in the spectrum of the associated operator (1.1).

In the case of quasiperiodic and soliton solutions, one obtains compact and noncompact Riemann surfaces (see for example Alber and Marsden (1992, 1994) and sections 3 and 4 of this paper). The $\mu$-representation yields complex angle representations on Jacobi varieties in a way described in Alber et al (1994, 1995). This yields, amongst other things, a representation of the $\mu$-variables in terms of $\theta$ and $\tau$-functions (see McKean 1979, Mumford 1983). In the last step, the solution of the initial nonlinear equation is expressed in terms of the $\mu$-variables. 
In the $\mu$-representation the calculation of modulation equations is facilitated. Whitham equations can be obtained in terms of holomorphic and meromorphic differentials. They describe the slow modulation of branch points on the Riemann surfaces (see Flaschka et al 1980). Notice that the monodromy effect to be described in section 4 is important in investigating Whitham equations of $N$-component systems.

In the most general case, the $\mu$-representation describes a geodesic flow for the metric $\mathrm{d} s^{2}=\sum_{j=1}^{n} g_{j j} \mu^{j^{2}}$ on complex Riemannian manifolds. Classification of all such metrics which yield separation of variables in Hamilton-Jacobi equations and integrability of the geodesic flows has been investigated recently in Benenti et al (1995). In the context of their paper $\mu$-variables are viewed as generalized elliptic coordinates.

We will use this interpretation of the $\mu$-variables in the next section to clarify a connection to mechanical systems. Namely, the procedure outlined in this section also produces many important classical as well as new integrable mechanical systems with Hamiltonians of the form (2.10) for particular choices of $B$ and $V$. These expansions can also be used to obtain semiclassical solutions of the eigenvalue problem represented by $L$ in (1.1) and yields asymptotics for the Bloch eigenfunctions. These finite dimensional representations also permit the analysis of a connection between caustic envelopes in the phase space of these mechanical systems and Maslov-Keller indices in the semiclassical approximation of the spectrum of the Laplace-Beltrami operators (see Alber $(1989,1991)$, Alber and Marsden (1992) and sections 6 and 7 of this paper).

\section{The family of homoclinic orbits described as Hamiltonian flows on a Riemann surface}

Here we recall the $\mu$ representation for the quasiperiodic and homoclinic Hamiltonian flows of the classical Neumann problem of mechanics.

Devaney (1978) investigated homoclinic orbits of the Neumann problem. Moser (1981) studied equilibrium solutions possessing stable and unstable manifolds for this mechanical problem in connection with the spectral theory of finite gap and reflectionless potentials of Schrődinger operators and quasiperiodic and soliton solutions of the KdV equation. In particular, it was shown that equilibrium solutions are associated with the reflectionless potentials which correspond to a set of negative double points of the discrete spectrum.

Here we show that a special limiting process applied to the quasiperiodic $\mu$ representation leads to the introduction of a new set of first integrals and exponential Hamiltonians for homoclinic orbits (see Alber and Marsden 1994 for details). This approach will be applied in the next section to the 2-component Dym system to describe special classes of solutions with monodromy.

The system of first integrals for the Neumann problem, namely

$$
\begin{aligned}
& F_{j}(y, \dot{y}): T S^{n} \rightarrow \boldsymbol{R} \\
& F_{j}(y, \dot{y})=y_{j}^{2}+\frac{1}{2} \sum_{k \neq j} \frac{\left(\dot{y}_{j} y_{k}-\dot{y}_{k} y_{j}\right)^{2}}{l_{k}^{2}-l_{j}^{2}} \quad j=1, \ldots, n
\end{aligned}
$$

plays a central role in Devaney's (1978) description of transversal homoclinic orbits. Here

$$
l_{0}<l_{1}<\cdots<l_{n} \quad \text { and } \quad l_{0}=0 .
$$

Namely, he proved that the first integrals are identically zero along the orbits; i.e.

$$
F_{j}(y, \dot{y})=0 \quad j=1, \ldots, n .
$$


We shall use this observation together with the $\mu$ representations for the quasiperiodic flows of the Neumann problem, i.e. solutions of the system

$$
\frac{\partial \mu_{j}}{\partial x}=2 \frac{\sqrt{-\prod_{r=1}^{n}\left(\mu_{j}-m_{r}\right) \prod_{k=1}^{n+1}\left(\mu_{j}-a_{k}\right)}}{\prod_{i \neq j}\left(\mu_{j}-\mu_{i}\right)} \quad j=1, \ldots, n .
$$

In accordance with the general result described in section 2, this system is defined on the symmetric product

$$
\Gamma:(\Re \times \cdots \times \Re) / \sigma_{n}
$$

of $n$ copies of the Riemannian surface

$$
\Re: P^{2}=-\prod_{k=1}^{n+1}\left(\mu-a_{k}\right) \prod_{r=1}^{n}\left(\mu-m_{r}\right)
$$

in fact, $\Gamma$ is a Lagrangian submanifold of the phase space $C^{2 n}$. In this setting, the first integrals of the problem can be represented in the form

$$
F_{j}=\frac{\prod_{r=1}^{n}\left(a_{j}-m_{r}\right)}{\prod_{k \neq j}\left(a_{j}-a_{k}\right)} .
$$

Here,

$$
a_{j}=-2 l_{j}^{2} \quad j=0, \ldots, n
$$

and the constants $m_{r}$ and $a_{j}$ are the endpoints of the gaps in the spectrum of the associated Schrödinger equation. Condition (3.3) and formula (3.7) yield the following choice of first integrals $m_{j}$ :

$$
m_{j}=a_{j}=b_{j} \quad j=1, \ldots, n, a_{0}=0
$$

which corresponds to a homoclinic orbit, meaning that all roots of the basic polynomial of the Riemannian surface (3.6) are double negative roots, except $a_{0}=0$.

The system (3.4) corresponding to the case of a singular spectrum (3.8) coincides with the system of equations describing profiles of the soliton solutions of the KdV equation; that is, with

$$
\frac{\partial \mu_{j}}{\partial x}=2 \frac{\sqrt{-\mu_{j}} \prod_{k=1}^{n}\left(\mu_{j}-b_{k}\right)}{\prod_{i \neq j}\left(\mu_{j}-\mu_{i}\right)} \quad j=1, \ldots, n .
$$

This system may be rewritten as

$$
\frac{\partial \mu_{j} / \partial x}{\left(\mu_{j}-b_{r}\right)}=2 \frac{\sqrt{-\mu_{j}} \prod_{k \neq r}^{n}\left(\mu_{j}-b_{k}\right)}{\prod_{i \neq j}\left(\mu_{j}-\mu_{i}\right)} \quad j=1, \ldots, n, r=1, \ldots, n .
$$

Summing with respect to $j=1, \ldots, n$ for each $r$ and integrating, one obtains the following angle variables

$$
\theta_{r}=\sum_{j=1}^{n} \frac{1}{2} \int_{\mu_{j}^{0}}^{\mu_{j}} \frac{\mathrm{d} \mu_{j}}{\left.\sqrt{-\mu_{j}}\left(\mu_{j}-b_{r}\right)\right)}=x+\theta_{r}^{0} \quad r=1, \ldots, n .
$$

The corresponding action function and action variables can be chosen as follows

$$
S=\sum_{j=1}^{n} \frac{1}{2} \int_{\mu_{j}^{0}}^{\mu_{j}} \frac{\log \left(\mu_{j}-b_{r}\right) \mathrm{d} \mu_{j}}{\sqrt{-\mu_{j}}} \quad I_{r}=a_{r}, r=1, \ldots, n .
$$


This results in $\theta_{r}=-\partial S / \partial I_{r}$ and so leads to the following form of the first integrals:

$$
P_{j}=\frac{\sum_{k=1}^{n} \log \left(\mu_{j}-b_{k}\right)}{2 \sqrt{-\mu_{j}}} \quad j=1, \ldots, n .
$$

This gives a new Hamiltonian on $C^{2 n}$ that is of exponential type for the system (3.9) describing the family of homoclinic orbits, namely

$$
H=\frac{\sum_{j=1}^{n}\left(\mathrm{e}^{2 \sqrt{-\mu_{j}} P_{j}}-\bar{C}\left(\mu_{j}\right)\right)}{\prod_{r \neq j}\left(\mu_{j}-\mu_{r}\right)}
$$

where $\bar{C}(\mu)=\prod_{k=1}^{n}\left(\mu-a_{k}\right)$. Note that the flow of (3.14) is defined on the noncompact level sets of (3.13) and it is linearized in terms of the angle variables (3.11).

The homoclinic angle representations (3.11) are defined on a noncompact Jacobi variety $\mathcal{J}$. The variety $\mathcal{J}$ is defined by a generalized Abel-Jacobi map (3.11), as in Ercolani (1989). This map is associated with $\Re^{n}$, the symmetric product of $n$ copies of the Riemann surface

$$
\Re: P=\frac{1}{2 \sqrt{-\mu} \prod_{r=1}^{n}\left(\mu-b_{r}\right)} .
$$

Let the double covering of $\Re^{n}$ be denoted $\widetilde{\Re^{n}}$. This covering is defined by the following change of variables

$$
\xi_{j}^{2}=-\mu_{j} \quad \beta_{j}^{2}=-b_{j} \quad j=1, \ldots, n .
$$

The Hamiltonian (3.14) defines a dynamical system on $\Re^{n}$, which lifts to a dynamical system on $\widetilde{\mathfrak{R}^{n}}$. An analysis of the angle representation (3.11) shows that the system has a homoclinic point $b=\left(b_{1}, \ldots, b_{n}\right)$ on $\Re^{n}$ as $x \rightarrow \infty$ (or $x \rightarrow-\infty$ ) and, correspondingly, two heteroclinic points $\beta^{+}$and $\beta^{-}$on $\widetilde{\Re^{n}}$ associated with the following values of $\mu_{j}$ :

$$
\mu_{j}=b_{j} \quad j=1, \ldots, n .
$$

(for details see Alber and Marsden 1994). The stable $W^{s}$ (and unstable $W^{u}$ ) manifolds of the point $b$ are coincident and consist of the orbits in $\Re^{n}$ that are forward (and backward) asymptotic to the homoclinic point $b$. On the other hand, the unstable manifold of the point $\beta^{+}$connects it to the point $\beta^{-}$in $\widetilde{\mathfrak{R}^{n}}$ and similarly for the stable manifold; these heteroclinic manifolds cover the homoclinic manifold in $\Re^{n}$.

\section{Solutions with monodromy for the 2-component Dym system}

For the Neumann problem, the spectral parameter appears linearly in the potential of the corresponding Schrödinger equation. However, in other systems of interest, this dependence is more complicated. In this section we discuss such systems; specifically, we consider Hamiltonian systems on Riemann surfaces that are associated with energydependent Schrödinger operators. In these systems, the dependence of the potential on the spectral parameter can have a pole. We also consider $N$-component systems of equations, using the hierarchy of the 2-component Dym systems as an example. In particular, we construct special solutions with monodromy for these systems of equations.

Using the method described in sections 2 and 3 with a potential of the form

$$
V=u(x, t)+\lambda+\frac{v(x, t)}{\lambda}
$$

and the dynamical recurrence chain for coefficients $b_{j}$ obtained from the dynamical generating equation (2.3) by setting $B(x, \lambda)=b_{0}(x) \lambda+b_{1}(x)$ and equating coefficients 
of the same power of $\lambda$, one obtains the following system of coupled PDEs which is called the first member of the hierarchy of the 2-component Dym systems,

$$
\begin{aligned}
& \frac{\partial u}{\partial t}=\frac{1}{4} u^{\prime \prime \prime}-\frac{3}{2} u u^{\prime}+v^{\prime} \\
& \frac{\partial v}{\partial t}=-u^{\prime} v-\frac{1}{2} u v^{\prime} .
\end{aligned}
$$

Notice that the choice of polynomial $B_{l}(x, \lambda)=b_{0}(x) \lambda^{l}+\cdots+b_{l}$ of the $l$ th order yields the $l$ th integrable system of evolution equations of the 2-component Dym hierarchy.

In what follows, we will introduce a Hamiltonian structure for the set of quasiperiodic solutions of the equations from the hierarchy of 2-component Dym systems. Certain limiting procedures applied to this Hamiltonian system will then yield special solutions with monodromy.

One obtains $\mu$-representations for the $l$ th system from the hierarchy after substituting $E=\mu_{j}$ into the generating equations (2.4) and (2.5):

$$
\begin{array}{ll}
\mu_{j}^{\prime}=\frac{1}{\prod_{r \neq j}^{n}\left(\mu_{j}-\mu_{r}\right)} \sqrt{\frac{C\left(\mu_{j}\right)}{\mu_{j}}} \quad j=1, \ldots, n \\
\dot{\mu}_{j}=\frac{B_{l}\left(\mu_{j}\right)}{\prod_{r \neq j}^{n}\left(\mu_{j}-\mu_{r}\right)} \sqrt{\frac{C\left(\mu_{j}\right)}{\mu_{j}}} \quad j=1, \ldots, n
\end{array}
$$

where

$$
C\left(\mu_{j}\right)=-L_{0}^{2} \prod_{k=1}^{2 n+2}\left(\mu_{j}-m_{k}\right)
$$

$l$ labels the number of a system in the 2-component Dym hierarchy, and $L_{0}$ is a constant. In the case $l=1$ one obtains $\mu$-representations for the system (4.1). Each of the $\mu$-variables is defined on a copy of the Riemann surface

$$
\Re: P^{2}=\frac{C(\mu)}{\mu} .
$$

Recall that the $\mu$ variables move along cycles on the corresponding Riemann surface (4.4) over the basic cuts between $m_{2 j}$ and $m_{2 j-1}, j=1, \ldots, n$.

Equations (4.2) and (4.3) define Hamiltonian systems with the Hamiltonians

$$
H=\sum_{j=1}^{n} \frac{D\left(\mu_{j}\right)\left(P_{j}^{2}-C\left(\mu_{j}\right) / \mu_{j}\right)}{\prod_{r \neq j}^{n}\left(\mu_{j}-\mu_{r}\right)}
$$

where $D\left(\mu_{j}\right)=1$ and $D\left(\mu_{j}\right)=B_{l}\left(\mu_{j}\right)$ in the stationary (4.2) and dynamical (4.3) cases, respectively. These have the following common set of first integrals

$$
P_{j}^{2}=\frac{C\left(\mu_{j}\right)}{\mu_{j}} \quad j=1, \ldots, n .
$$

Now we construct a system of angle variables to linearize quasiperiodic Hamiltonian flows using a method similar to the one described in section 3 .

Here we are dealing with a degenerate system because the genus of the associated Riemann surface is $(n+1)$ and yet we have only $n \mu$-variables. Related to this, there is also a degeneracy in the problem of inversion. 
Namely, after rearranging the systems of equations (4.2) and (4.3), summing and using Lagrange-type interpolation formulae, one obtains the following expressions

$$
\begin{aligned}
& \sum_{j=1}^{n} \frac{\mu_{j}^{k} \mu_{j}^{\prime}}{\sqrt{C\left(\mu_{j}\right) \mu_{j}}}=\sum_{j=1}^{n} \frac{\mu_{j}^{k}}{\prod_{r \neq j}^{n+1}\left(\mu_{j}-\mu_{r}\right)}=\delta_{k, 1}^{n-1} \\
& \sum_{j=1}^{n} \frac{\mu_{j}^{k} \dot{\mu}_{j}}{\sqrt{C\left(\mu_{j}\right) \mu_{j}}}=\sum_{j=1}^{n} \frac{\mu_{j}^{k} B_{2}\left(\mu_{j}\right)}{\prod_{r \neq j}^{n+1}\left(\mu_{j}-\mu_{r}\right)}=\delta_{k, 2}^{n-2}
\end{aligned}
$$

where $k=1, \ldots, n$ and $\delta_{j}^{i}$ is the Kronecker delta. After integrating (4.7), one obtains the angle variables:

$\theta_{k}=\sum_{j=1}^{n} \int_{\mu_{j}^{0}}^{\mu_{j}} \frac{\mu_{j}^{k} \mathrm{~d} \mu_{j}}{\sqrt{C\left(\mu_{j}\right) \mu_{j}}}=\delta_{k, 1}^{n-1} x+\delta_{k, 2}^{n-2} t+\theta_{k}^{0}, \quad I_{k}=c_{2 n-k+1}, k=1, \ldots, n$

where $c_{2 n-k+1}$ are coefficients of the polynomial $C(\mu)$ and $\theta_{k}^{0}$ are constants. The above integrals are taken along cycles $a_{j}$ over basic cuts on the Riemann surface

$$
\Re: W^{2}=C(\mu) \mu
$$

which has genus $g=n+1$. The system (4.8) is equivalent to a degenerate Jacobi problem of inversion. This degeneracy can be resolved by solving first the problem of inversion in terms of Riemann $\Theta$ functions on the $(n+1)$-dimensional Jacobian and then projecting on the $n$-dimensional subvariety. (For details of the Jacobi problem of inversion see for example Ercolani and McKean (1990) and Alber and Alber (1987).)

On the other hand, the monodromy effect can be described as follows. Setting $m_{2 n+2}=m_{2 n+1}=b$ in the initial Hamiltonian systems (4.2) and (4.3) yields in the manner described above, a system of angle variables

$\theta_{k}=\sum_{j=1}^{n} \int_{\mu_{j}^{0}}^{\mu_{j}} \frac{\mu_{j}^{k+1} \mathrm{~d} \mu_{j}}{\sqrt{\bar{C}\left(\mu_{j}\right) \mu_{j}}}=\sigma_{k, 1} x+\sigma_{k, 2} t+\theta_{k}^{0} \quad k=1, \ldots, n-1$

$\theta_{n}=\sum_{j=1}^{n} \int_{\mu_{j}^{0}}^{\mu_{j}} \frac{\mu_{j}^{k+1} \mathrm{~d} \mu_{j}}{\left(\mu_{j}-b\right) \sqrt{\bar{C}\left(\mu_{j}\right) \mu_{j}}}=\theta_{n}^{0}$

where $\bar{C}\left(\mu_{j}\right)=-\prod_{k=1}^{2 n}\left(\mu-m_{k}\right)$ and $\sigma_{k, 1}, \sigma_{k, 2}$ are constants. This can be viewed as a problem of inversion with a singularity at $\mu=b$, resulting in a phenomenon of monodromy for the Hamiltonian system.

We define a Jacobian fibration with the base parametrized by roots of the basic polynomial $C(\lambda) \lambda$ of the Riemann surface (4.9). Now, moving $b$ along a certain closed loop in the space of parameters (the base of the fibration) can lead to a nontrivial shift in the action angle variables.

We demonstrate this using the $x$-flow. Consider first the case $n=1$. The limiting process $m_{3}, m_{4} \rightarrow b$ yields the following angle variable

$$
\theta_{1}=\int_{\mu_{1}^{0}}^{\mu_{1}} \frac{\mu_{1} \mathrm{~d} \mu_{1}}{\left(\mu_{1}-b\right) \sqrt{-\mu_{1}\left(\mu_{1}-m_{1}\right)\left(\mu_{1}-m_{2}\right)}}=L_{0} x+\theta_{1}^{0} .
$$

In the case of $n=2$, the limiting angle variables are as follows

$$
\begin{aligned}
& \theta_{1}=-\frac{\partial S}{\partial \beta_{2}}=\int_{\mu_{1}^{0}}^{\mu_{1}} \frac{\mu_{1} \mathrm{~d} \mu_{1}}{\sqrt{\bar{C}_{4}\left(\mu_{1}\right) \mu_{1}}}+\int_{\mu_{2}^{0}}^{\mu_{2}} \frac{\mu_{2} \mathrm{~d} \mu_{2}}{\sqrt{\bar{C}_{4}\left(\mu_{2}\right) \mu_{2}}}=L_{0} x+\theta_{1}^{0} \\
& \theta_{2}=-\frac{\partial S}{\partial \beta_{1}}=\int_{\mu_{1}^{0}}^{\mu_{1}} \frac{\mu_{1} \mathrm{~d} \mu_{1}}{\left(\mu_{1}-b\right) \sqrt{\bar{C}_{4}\left(\mu_{1}\right) \mu_{1}}}+\int_{\mu_{2}^{0}}^{\mu_{2}} \frac{\mu_{2} \mathrm{~d} \mu_{2}}{\left(\mu_{2}-b\right) \sqrt{\bar{C}_{4}\left(\mu_{2}\right) \mu_{2}}}=\theta_{2}^{0}
\end{aligned}
$$


where

$$
S=\int_{\mu_{1}^{0}}^{\mu_{1}} \frac{\sqrt{\bar{C}_{4}\left(\mu_{1}\right) \mu_{1}} \mathrm{~d} \mu_{1}}{\mu_{1}-b}+\int_{\mu_{2}^{0}}^{\mu_{2}} \frac{\sqrt{\bar{C}_{4}\left(\mu_{2}\right) \mu_{2}} \mathrm{~d} \mu_{2}}{\mu_{2}-b}
$$

is an action function and

$$
\bar{C}_{4}(\mu)=-\left(\mu-m_{1}\right)\left(\mu-m_{2}\right)\left(\mu-m_{3}\right)\left(\mu-m_{4}\right) .
$$

The variables $\mu_{1}$ and $\mu_{2}$ move along cycles $a_{1}$ and $a_{2}$ over the cuts $\left[m_{1}, m_{2}\right]$ and $\left[m_{3}, m_{4}\right]$ on the Riemann surface $W^{2}=\mu \bar{C}_{4}(\mu)$. There is also a singularity at $\mu=b$. Transport of a system of canonical action-angle variables, which linearize the Hamiltonian flow, along a certain loop in the space of parameters $\left(b, m_{j}\right)$ in a way similar to the case of the spherical pendulum and some other integrable systems with monodromy (see, for example, Duistermaat 1980, Bates and Zou 1993) will result in a nontrivial shift, which is a manifestation of the monodromy phenomenon. This can be demonstrated as follows. Canonical actions are calculated in terms of periods of the differential

$$
I_{j}=\oint_{a_{j}} \mathrm{~d} S=\oint_{a_{j}} \frac{\sqrt{\bar{C}_{4}(\mu) \mu} \mathrm{d} \mu}{\mu-b}
$$

along cycles $a_{j}$ on the Riemann surface; for details see Arnold (1978). Now suppose initially that $b$ does not belong to any of the cycles $a_{1}$ and $a_{2}$. Then we move $b$ along a closed loop on the Riemann surface around one of the branch points $m_{j}$. At some moment $b$ becomes a branch point itself, which results in a shift of the action variable that is given by the residue of the integrand at $\mu=b$.

Flaschka et al (1980) proposed the use of $\mu$-representations in calculations of modulation equations and showed that, in the case of the KdV equation, Whitham theory can be described in terms of holomorphic and meromorphic differentials on the moduli spaces of Riemann surfaces. Namely, they described the slow modulation of branch points of the Riemann surfaces.

The monodromy effect described in this section provides an example of a singularity of Whitham-type equations. It has to be taken into account in the investigation of modulational theory of the $N$-component systems.

\section{Mechanical systems and nonlinear equations}

In section 2 we recalled the link between quasiperiodic solutions of integrable nonlinear equations and Hamiltonian systems on Riemann surfaces established using generating equations and algebraic-geometric methods. Here we expand on this by generating the Euler-Lagrange equations for some well known mechanical problems. This establishes a link between such systems and the stationary Hamiltonian flows for classes of solutions of integrable nonlinear PDEs. (See, for example, Alber et al $(1994,1995)$ for additional information.) This will be used in section 6 to study the semiclassical theory of such solutions.

One obtains the Euler-Lagrange equations, using the method of generating equations described in section 2 and by changing variables in $B(x, \lambda)$ from $\mu$ 's to $q$ 's:

$$
B(x, \lambda)=\prod_{i=1}^{n}\left(\lambda-\mu_{i}(x, t)\right)=\sum_{j=1}^{n+1} \prod_{r=1, r \neq j}^{n+1}\left(\lambda-l_{r}\right) q_{j}^{2}(x) .
$$


We also denote

$$
W(\lambda)=\frac{C(\lambda)}{K(\lambda)}=\frac{\prod_{r=j}^{2 n+N}\left(\lambda-l_{j}\right)}{\prod_{j=r}^{M}\left(\lambda-a_{r}\right)}
$$

where $\lambda$ is a complex parameter and the constants $l_{j}$ and $a_{r}$ will be shown to be determined by the parameters and first integrals of the particular mechanical system.

The expression (5.1) can be viewed as a definition of generalized elliptic $\mu$ coordinates on Riemann surfaces. Coordinates $q_{j}^{2}=x_{j}^{2} / l_{j}$ are normalized Cartesian coordinates of a particular mechanical system. Equating coefficients for the $\lambda^{n}$ in (5.1) yields

$$
\sum_{j=1}^{n+1} q_{j}^{2}=\sum_{j=1}^{n+1} \frac{x_{j}^{2}}{l_{j}}=1
$$

The parameters $l_{j}$ can be complex and so (5.2) defines a pseudo-sphere in $\boldsymbol{C}^{n+1}$ and provides a constraint for the Euler-Lagrange equations.

In terms of the $q$-variables, our mechanical problems may be thought of as problems of geodesics on the pseudosphere (5.2) in the presence of a potential. In section 6 we link them to the Laplace-Beltrami operators in a way similar to the method of geometric optics and study semiclassical approximations for eigenfunctions of Laplace-Beltrami operators having the form of complex WKB modes.

Substituting $B$ and $V$ into (2.4) and setting $\lambda=l_{r}$ one by one, a system of equations is generated for the functions $q_{j}(x)$. Below we obtain several different integrable systems for different choices of the potential $V(x, \lambda)$.

Recall (from Moser 1981) the classical Neumann and Jacobi problems. The Neumann problem for the motion of a particle on the $n$-sphere in the field of a quadratic potential corresponds to $V=u(x)-\lambda$. Substituting (5.1) into (2.4), and setting $\lambda=l_{r}$ one by one, yields a system of Euler-Lagrange equations,

$$
q_{j}^{\prime \prime}-q_{j}\left(u-l_{j}\right)=0 \quad j=1, \ldots, n+1
$$

corresponding to the Lagrangian

$$
L=\sum_{j=1}^{n+1} q_{j}^{\prime 2}+u\left(\sum_{j=1}^{n+1} q_{j}^{2}-1\right)-\sum_{j=1}^{n+1} l_{j} q_{j}^{2} .
$$

Notice that the Euler-Lagrange equations and constraint $\sum_{j=1}^{n+1} q_{j}^{2}=1$ yield the following expression for $u$ :

$$
u(x)=\sum_{j=1}^{n+1} l_{j} q_{j}^{2}-\sum_{j=1}^{n+1} q_{j}^{\prime 2}
$$

The function $u$ plays the role of Lagrange multiplier.

For the Jacobi problem of geodesics on $n$-dimensional quadrics (free motion on quadrics), one takes

$$
V=\frac{u(x)}{\lambda}
$$

and the Euler-Lagrange equations are

$$
q_{j}^{\prime \prime}-q_{j} \frac{u}{l_{j}}=0
$$


where $l_{j}$ are semiaxes of the ellipsoid. Here the Lagrangian and $u$ are as follows

$$
L=\sum_{j=1}^{n+1} l_{j} q_{j}^{\prime 2}+u\left(\sum_{j=1}^{n+1} q_{j}^{2}-1\right) \quad u(x)=\frac{\sum_{j=1}^{n+1} q_{j}^{\prime 2}}{\sum_{j=1}^{n+1} \frac{q_{j}^{2}}{l_{j}}} .
$$

Next we consider a linear combination of the potentials for the Neumann and Jacobi problems. (Mechanical systems associated with this potential were discussed in Braden (1982).) Here we are interested in the connection between this problem and the spatial flow for a particular class of solutions of the system of coupled PDEs (4.1). Here, $V=u(x)+\lambda+v(x) / \lambda$ and the Euler-Lagrange equations are

$$
q_{j}^{\prime \prime}-q_{j}\left(u+l_{j}+\frac{v}{l_{j}}\right)=0
$$

with the constraint $\sum_{j=1}^{n+1} q_{j}^{2}=1$. The Lagrangian and the potential are as follows:

$$
\begin{aligned}
& L=\sum_{j=1}^{n+1} q_{j}^{\prime 2}+u\left(\sum_{j=1}^{n+1} q_{j}^{2}-1\right)+\sum_{j=1}^{n+1} l_{j} q_{j}^{2}-\frac{1}{\sum_{j=1}^{n+1} \frac{q_{j}^{2}}{l_{j}}} \\
& u(x)=-\sum_{j=1}^{n+1} q_{j}^{\prime 2}-\sum_{j=1}^{n+1} l_{j} q_{j}^{2}+\frac{1}{\sum_{j=1}^{n+1} \frac{q_{j}^{2}}{l_{j}}} .
\end{aligned}
$$

Here we are using

$$
v(x)=\frac{1}{\left(\sum_{j=1}^{n+1} \frac{q_{j}^{2}}{l_{j}}\right)^{2}}
$$

which is obtained from a recurrence chain generated by (2.4).

Now consider the flow for the Camassa-Holm shallow-water equation (see Alber et al 1994). Here

$$
V=1+\frac{u(x)}{\lambda}
$$

and

$$
q_{j}^{\prime \prime}-q_{j}\left(\frac{u}{l_{j}}+1\right)=0 .
$$

The Lagrangian and potential are as follows

$$
L=\sum_{j=1}^{n+1} q_{j}^{\prime 2}+u\left(\sum_{j=1}^{n+1} q_{j}^{2}-1\right)-\frac{1}{\sum_{j=1}^{n+1} \frac{q_{j}^{2}}{l_{j}}} \quad u(x)=-\sum_{j=1}^{n+1} q_{j}^{\prime 2}+\frac{1}{\sum_{j=1}^{n+1} \frac{q_{j}^{2}}{l_{j}}} .
$$

The next example is provided by the spatial flow for a generalization of the shallowwater equation. Calogero and Degasperis (1982) studied an interesting new class of integrable mechanical systems. We generalize this class by considering a 2-component case whose associated system of coupled PDEs has the form of a coupled system of equations of shallow-water type. Here,

$$
V=u(x)+\frac{v(x)}{\lambda}
$$

and

$$
q_{j}^{\prime \prime}-q_{j}\left(u+\frac{v}{l_{j}}\right)=0
$$


The Lagrangian and potential are as follows

$$
L=\sum_{j=1}^{n+1} l_{j} q_{j}^{\prime 2}+v\left(\sum_{j=1}^{n+1} q_{j}^{2}-1\right)-\frac{u}{\sum_{j=1}^{n+1} \frac{q_{j}^{2}}{l_{j}}} \quad u(x)=-\sum_{j=1}^{n+1} q_{j}^{\prime 2}+\frac{1}{\sum_{j=1}^{n+1} \frac{q_{j}^{2}}{l_{j}}} .
$$

See the appendix for the mechanical system associated with the 2-component $\mathrm{KdV}$ system.

\section{Complex semiclassical solutions}

Recall that solutions of integrable systems can be reduced to finite-dimensional Hamiltonian systems with the commuting $x$ and $t$ flows as outlined in section 2. Hamiltonian flows for a variety of important integrable mechanical systems are constructed using the same techniques and are linked to the spatial flows for particular classes of solutions of the nonlinear PDEs.

Each of these flows can be characterized by a Hamiltonian of the form (2.10). Here we discuss the semiclassical limits of the associated Laplace-Beltrami operators for these systems. In what follows, we recall from Alber (1989, 1991) and Alber and Marsden (1992), a method of complex geometric asymptotics for integrable Hamiltonian flows on Riemann surfaces. We will use geometric asymptotics to describe the quantization conditions of Bohr-Sommerfeld-Keller (BSK) type. Then we will investigate the dependence of these conditions on the parameters (i.e. the first integrals) of the system. This dependence near singularities produces effects caused by the classical and semiclassical monodromy.

Let us consider quadratic complex Hamiltonians of the form (2.10) defined on $\boldsymbol{C}^{2 n}$. We think of $C^{2 n}$ as being the cotangent bundle of $C^{n}$, with configuration variables $\mu_{1}, \ldots, \mu_{n}$ and with canonically conjugate momenta $P_{1}, \ldots, P_{n}$.

Notice that the Hamiltonians for quasiperiodic solutions of the systems considered in the previous sections are indeed of this form. We consider the functions $g^{j j}$ as components of a (diagonal) Riemannian metric, construct the associated Laplace-Beltrami operator, and then the stationary Schrödinger equation

$$
\nabla^{j} \nabla_{j} U+w^{2}(E-V) U=0
$$

defined on $\boldsymbol{C}^{n}$. Here $\nabla^{j}$ and $\nabla_{j}$ are covariant and contravariant derivatives defined by the tensor $g^{j j}$ and $w$ (which is the inverse of Planck's constant $\hbar$ ) and $E$ (the energy eigenvalue) are parameters. Note also that in general, the metric tensor is not constant, and even may have singularities, so that the kinetic term in the expression for $H$ is not purely quadratic.

Now we establish a link between (6.1) and the Hamiltonian system (2.10) by means of geometric asymptotics; namely, we consider the following function that is similar to the well known ansatz from WKB theory:

$$
\begin{aligned}
U\left(z_{1}, \ldots, z_{n}\right) & =\sum_{k} A_{k}\left(\mu_{1}, \ldots, \mu_{n}\right) \exp \left[\mathrm{i} w S_{k}\left(\mu_{1}, \ldots, \mu_{n}\right)\right] \\
& =\sum_{k} \prod_{j=1}^{n} U_{k j}\left(\mu_{j}\right)=\sum_{k} \prod_{j=1}^{n}\left(A_{k j}\left(\mu_{j}\right) \exp \left[\mathrm{i} w S_{k j}\left(\mu_{j}\right)\right]\right)
\end{aligned}
$$

which is a multivalued function of several complex variables defined on $\boldsymbol{C}^{n}$. If, instead, one considers $U$ to be defined on the covering space of the Jacobi variety of the problem, then $U$ becomes single valued. The functions present in this expression together with $r$, which denotes a vector of Maslov indices, will be determined below. Note that $k$ also labels the classical paths between initial and current points in the configuration space. 
Substituting (6.2) in (6.1), equating coefficients for $w$ and $w^{2}$ and integrating, we obtain the amplitude function $A$, which is a solution of the transport equation, in the form

$$
A=\frac{A_{0}}{\sqrt{(D \operatorname{det} J)}}
$$

where $D=\sqrt{\prod_{l=1}^{n} g_{l l}}$ is the volume element of the metric and $J$ is the Jacobian of the change of coordinates from the $\mu$-representation to the angle $\theta$-representation. We also find that the phase function $S$ is a solution of the Hamilton-Jacobi equation

$$
\Delta^{j} S \Delta_{j} S-V=E
$$

so that it coincides with the action function. Finally, we describe the dependence of the WKB modes (6.2) on $t$ as a parameter, by introducing commuting $t$-flow for the $\mu$ variables described by (2.7).

\section{Semiclassical monodromy}

Now we can apply the constructions that have been developed above to the case of the special class of solutions of the 2-potential Dym system for $n=2$ (see section 4). The Hamiltonian and complex geometric asymptotic solution in this case have the form:

$$
H=\frac{1}{2} \frac{P_{1}^{2}-\frac{C_{5}\left(\mu_{1}\right)}{\mu_{1}-b}}{\mu_{1}-\mu_{2}}+\frac{1}{2} \frac{P_{2}^{2}-\frac{C_{5}\left(\mu_{2}\right)}{\mu_{2}-b}}{\mu_{2}-\mu_{1}} .
$$

and

$U=\sum_{k=\left(k_{1}, k_{2}\right)} A_{0} \frac{\sqrt{\mu_{1} \mu_{2}}}{\left(\left(\mu_{1}-b\right)\left(\mu_{2}-b\right)\right)^{\frac{1}{2}}\left(C_{5}\left(\mu_{1}\right)\left(C_{5}\left(\mu_{1}\right)\right)^{\frac{1}{4}}\right.} \exp \left[\mathrm{i} w S_{k 1}\left(\mu_{1}\right)+S_{k 2}\left(\mu_{2}\right)\right]$

where

$$
\begin{aligned}
& S_{k 1}\left(z_{1}\right)=\int_{\mu_{1}^{0}}^{\mu_{1}} \frac{\sqrt{C_{5}\left(\mu_{1}\right)} \mathrm{d} \mu_{1}}{\mu_{1}-b}+k_{1} T_{1}+\frac{r_{1} \pi}{4} \\
& S_{k 2}\left(z_{2}\right)=\int_{\mu_{2}^{0}}^{\mu_{2}} \frac{\sqrt{C_{5}\left(\mu_{2}\right)} \mathrm{d} \mu_{2}}{\mu_{2}-b}+k_{2} T_{2}+\frac{r_{2} \pi}{4}
\end{aligned}
$$

and where $r=\left(r_{1}, r_{2}\right)$ is a vector of Maslov indices and

$$
\begin{aligned}
& T_{1}=\oint_{a_{1}} \frac{\sqrt{C_{5}\left(\mu_{2}\right)} \mathrm{d} \mu_{2}}{\mu_{2}-b} \\
& T_{2}=\oint_{a_{2}} \frac{\sqrt{C_{5}\left(\mu_{2}\right)} \mathrm{d} \mu_{2}}{\mu_{2}-b} .
\end{aligned}
$$

The amplitude $A$ has singularities at the branch points $\mu_{1}=m_{1}, m_{2}, \mu_{2}=m_{3}, m_{4}$ and at an additional singular point $b$ on the associated Riemann surface. Each time a trajectory approaches one of these singularities, we continue it to complex $x$ and go around a small circle in the complex plane enclosing the singularity. This results in a phase shift $( \pm \mathrm{i} \pi / 2)$ of the phase function $S$, which is common in geometric asymptotics. The indices $k_{1}$ and $k_{2}$ keep track of the number of oriented circuits for $\mu_{1}$ and $\mu_{2}$ around $a_{1}$ and $a_{2}$. The complex mode (7.2) is defined on the covering space of the complex Jacobi variety. Note that in the real case, this complex mode is defined on the covering space of a real subtorus. Keeping this in mind, quantum conditions of BSK type can be imposed as conditions on the number 
of sheets of the covering space of the corresponding Riemann surface for each coordinate $\mu_{1}$ and $\mu_{2}$ :

$$
\begin{aligned}
& \frac{\pi}{2} r_{1}+w k_{1} T_{1}=2 \pi N_{1} \\
& \frac{\pi}{2} r_{2}+w k_{2} T_{2}=2 \pi N_{2} .
\end{aligned}
$$

Here $N_{1}, N_{2}$ are integer quantum numbers related to each other and to integer indices $k_{1}, k_{2}$ and $r_{1}, r_{2}$ as follows

$$
w=\frac{2 \pi N_{1}}{k_{1} T_{1}}=\frac{1}{k_{2} T_{2}}\left(2 \pi N_{2}-\frac{\pi r_{2}}{2}\right)
$$

which is an asymptotic formulae for the eigenvalues of the stationary Schrödinger equation (6.1). (For details about Maslov-Keller indices and quantum conditions see amongst others Robbins (1991) and Alber (1989, 1991).) Maslov-Keller indices play an important role in the numerical study of the semiclassical limit of the NLS equations presented in Jin et al (1994).

Notice that the quantum conditions (7.6) include a monodromy part after transport along a closed loop in the space of parameters.

\section{The stationary Schrödinger equation, Bloch functions and complex WKB solutions}

Lastly we contrast semiclassical approximations for the Bloch eigenfunctions to the approach of section 6 which uses a Riemannian metric associated with the complex Hamiltonian system associated with finite-dimensional solution space.

Namely we show how to obtain semiclassical approximations for the Bloch eigenfunctions of the operators defined in (1.3) and (1.4) and used in the inverse scattering transform theory (see Ablowitz and Segur 1981). The equation (1.3) is a natural generalization of a one-dimensional stationary Schrödinger equation

$$
-\frac{\hbar^{2}}{2 m} \frac{\partial^{2} \psi}{\partial x^{2}}+(V(x)-\lambda) \psi=0
$$

which we rewrite

$$
-\frac{\partial^{2} \psi}{\partial x^{2}}+V(x, \lambda, \epsilon) \psi=0
$$

where

$$
V(x, \lambda, E)=\frac{U(x, \lambda)}{\epsilon} \quad U(x, \lambda)=2 m(u(x)-\lambda)
$$

and $\epsilon=\hbar^{2}$. In our notation $\epsilon$ will be the complex parameter, and we will treat it independently from the energy $\lambda$. As usual, the WKB method is used to approximate $\psi$ for small values of the parameter $\epsilon$. In what follows we will use (8.2) to demonstrate semiclassical theory for the operators defined in (1.3).

We look for a solution of (8.2) in the form of a Bloch function

$$
\psi=\sqrt{\frac{B}{B_{0}}} \exp \left( \pm \int_{x_{0}}^{x} \frac{\sqrt{C}}{B} \mathrm{~d} x\right)
$$

where $B=B(x, \epsilon)$ is a function of $x$ and $\epsilon, C=C(\epsilon)$ is a function of $\epsilon$, and $B_{0}$ is a constant defined by the initial data. This gives a solution of (8.2) if and only if

$$
-B^{\prime \prime} B+\frac{B^{\prime 2}}{2}+2 B^{2} V=C
$$


as can be checked by direct substitution. This equation was first obtained in connection with the KdV equation in Gelfand and Dikey (1975) by investigating asymptotics of resolvents of the Sturm-Liouville equations.

Notice that (8.5) is just the stationary generating equation (2.4). We choose $B(x, \epsilon)$ in the form of a polynomial in $\epsilon$ and $C(\epsilon)$ to be a rational function with constant coefficients:

$$
B(x, \epsilon)=\sum_{l=0}^{n} b_{l}(x) \epsilon^{n-l} \quad C(\epsilon)=\frac{1}{\epsilon} \sum_{k=0}^{2 n} c_{k} \epsilon^{2 n-k} .
$$

The equation (8.5) with potential (8.3) coincides with the generating equation for the geodesic flow on an $n$-dimensional quadric. It was shown (see Cewen 1990, Alber et al 1994, 1995) to provide an $x$-flow for a partial differential equation of Dym type. The equation (8.5) yields a chain of recurrence relations between the coefficients $b_{l}$ and $c_{k}$ (for details see Alber et al 1994, 1995). In particular we have

$$
V=\frac{c_{2 n}}{b_{n}^{2}}
$$

and therefore

$$
b_{n}=\sqrt{\frac{c_{2 n}}{2 m(V-E)}} .
$$

As $\epsilon \rightarrow 0$, the functions $C(\epsilon)$ and $B(\epsilon)$ are asymptotic to $c_{2 n} / \epsilon$ and $b_{n}$. This yields the following asymptotics for the Bloch function

$$
\psi=\frac{\psi_{0}}{(u-E)^{\frac{1}{4}}} \exp \left( \pm \frac{\mathrm{i}}{\sqrt{\epsilon}} \int_{x_{0}}^{x} \sqrt{2 m(u-E)} \mathrm{d} x\right)
$$

which is precisely a well known WKB solution of the one-dimensional Schrödinger equation (8.1). A similar procedure produces asymptotics for Bloch functions corresponding to potentials with poles can be carried out using recurrence relations and geodesic flows on the associated Riemann surfaces. For example, a WKB solution in the case of the 2-potential Dym system described above has the form

$$
\psi=\frac{\psi_{0}}{v^{\frac{1}{4}}} \exp \left( \pm \frac{\mathrm{i}}{\sqrt{\epsilon}} \int_{x_{0}}^{x} \sqrt{2 m v} \mathrm{~d} x\right) .
$$

The Bloch function contains all necessary information about the finite gap spectrum and the associated Riemann surfaces. It is an ideal object for applying zero-dispersion limit theory. In the future, we intend to apply this approach to the semiclassical theory of (d)NLS and (f)NLS equations.

\section{Conclusions}

In this paper we have used so-called energy-dependent Schrödinger operators to establish a link between special classes of solutions of $N$-component systems of evolution equations and finite dimensional Hamiltonian systems on moduli spaces of Riemann surfaces. This yields in particular, a new class of solutions with monodromy for $N$-component systems of PDEs (equation (4.1)).

We also have used a connection between classes of solutions of nonlinear equations and finite dimensional mechanical systems to investigate the semiclassical theory of nonlinear equations. In the future, we intend to apply this approach for WKB-theory to the equations (1.3) and (1.4) in connection with the the zero-dispersion limit of $N$-component systems such as the coupled NLS equations. 


\section{Acknowledgments}

The research carried out by MSA was partially supported by NSF grants DMS 9403861 and 9508711. GGL gratefully acknowledges support from BRIMS, Hewlett-Packard Labs and from NSF DMS under grant 9508711 . The research carried out by JEM was partially supported by NSF grant DMS 9633161.

\section{Appendix. The spatial flow for the 2-component KdV system}

The discrete version of this system corresponds to the Toda and relativistic Toda lattices (for details see Alber 1987). Here

$$
V=\lambda^{2}+v(x) \lambda+u(x)
$$

and the Euler-Lagrange equations are

$$
q_{j}^{\prime \prime}-q_{j}\left(u+v l_{j}+l_{j}^{2}\right)=0
$$

The Lagrangian and potential are as follows

$$
\begin{aligned}
& L=\sum_{j=1}^{n+1} q_{j}^{\prime 2}+u\left(\sum_{j=1}^{n+1} q_{j}{ }^{2}-1\right)-\left(\sum_{j=1}^{n+1} l_{j} q_{j}{ }^{2}\right)^{2}-\sum_{j=1}^{n+1} l_{j}^{2} q_{j}{ }^{2} \\
& u(x)=-\sum_{j=1}^{n+1} q_{j}^{\prime 2}+\sum_{j=1}^{n+1} l_{j} q_{j}^{2}+\left(\sum_{j=1}^{n+1} l_{j} q_{j}{ }^{2}\right)^{2}+\sum_{j=1}^{n+1} l_{j}^{2} q_{j}{ }^{2} .
\end{aligned}
$$

Here we are using the expression

$$
v(x)=2 \sum_{j=1}^{n+1} l_{j} q_{j}^{2}
$$

which is obtained from the recurrence chain generated by (2.4) with $K=0$. Using the recurrence chain generated by the dynamical generating equation (2.4) and setting $B(x, \lambda)=b_{0}(x) \lambda+b_{1}(x)$ one obtains the following integrable system of coupled PDEs of $\mathrm{KdV}$ type:

$$
\begin{aligned}
& \frac{\partial u}{\partial t}=\frac{1}{4} v^{\prime \prime \prime}-v^{\prime} u-\frac{1}{2} v u^{\prime} \\
& \frac{\partial v}{\partial t}=u^{\prime}-\frac{3}{2} v v^{\prime} .
\end{aligned}
$$

\section{References}

Ablowitz M J and Clarkson P A 1991 Solitons, Nonlinear Evolution Equations and the Inverse Scattering Transform (Cambridge: Cambridge University Press)

Ablowitz M J and Segur H 1981 Solitons and the Inverse Scattering Transform (Philadelphia, PA: SIAM)

Alber S J 1991 Associated integrable systems J. Math. Phys. 32 916-22

Alber M S 1989 On integrable systems and semiclassical solutions of the stationary Schrödinger equations Inverse Problems 5 131-48

-1991 Hyperbolic geometric asymptotics Asymp. Anal. 5 161-72

Alber M S and Alber S J 1985 Hamiltonian formalism for finite-zone solutions of integrable equations C. R. Acad. Sci., Paris $301777-81$

—1987 Hamiltonian formalism for nonlinear Schrödinger equations and sine-Gordon equations J. Lond. Math. Soc. 36 176-92

Alber M S and Marsden J E 1992 On geometric phases for soliton equations Commun. Math. Phys. 149 217-40 
1994 Geometric Phases and Monodromy at Singularities (NATO ASI Series B 320) ed N M Ercolani et al (New York: Plenum) pp 273-96

-1996 Semiclassical monodromy and the spherical pendulum as a complex Hamiltonian system Fields Inst. Commun. 8 1-18

Alber M S, Camassa R, Holm D D and Marsden J E 1994 The geometry of peaked solitons and billiard solutions of a class of integrable PDEs Lett. Math. Phys. 32 137-51

1995 On the link between umbilic geodesics and soliton solutions of nonlinear PDE's Proc. R. Soc. A 450 677-92

Antonowicz M and Fordy A P 1987a A family of completely integrable multi-Hamiltonian systems Phys. Lett. 122A 95-9

—1987b Coupled KdV equations with multi-Hamiltonian structures Physica 28D 345-57

—1988 Coupled Harry Dym equations with multi-Hamiltonian structures J. Phys. A: Math. Gen. 21 L269-75

1989 Factorisation of energy dependent Schrödinger operators: Miura maps and modified systems Commun. Math. Phys. 124 465-86

Antonowicz M, Fordy A P and Liu Q P 1991 Energy-dependent third-order lax operators Nonlinearity 4 669-84

Arnold V I 1978 Mathematical Methods of Classical Mechanics (Berlin: Springer)

Bates L and Zou M 1993 Degeneration of Hamiltonian monodromy cycles Nonlinearity 6 313-35

Benenti S, Kalnins E G and Miller W 1995 Integrability, Stäckel spaces and rational potentials Preprint

Bronski J C and McLaughlin D W 1994 Semiclassical Behaviour in the NLS Equation: Optical Self-Focusing Instabilities, Singular Limits of Dispersive Waves (NATO ASI Series B 320) ed N M Ercolani et al (New York: Plenum) pp 21-38

Braden H 1982 A completely integrable mechanical system Lett. Math. Phys. 6 449-52

Calogero F and Degasperis A 1982 Spectral Transform and Solitons (Amsterdam: North-Holland)

Cewen C 1990 Stationary Harry-Dym's equation and its relation with geodesics on ellipsoid Acta Math. Sin. 6 $35-41$

Cushman R and Duistermaat J J 1988 The quantum spherical pendulum Bull. Am. Math. Soc. 19 475-9

Devaney R 1978 Transversal homoclinic orbits in an integrable system Am. J. Math. 100631

Duistermaat J J 1988 The quantum spherical pendulum Quantum Theories and Geometry ed M Cahen and M Flato (Dordrecht: Kluwer) pp 53-64

Ercolani N 1989 Generalized theta functions and homoclinic varieties Proc. Symp. Pure Appl. Math. 4987

Ercolani N and McKean H 1990 Geometry of KdV(4): Abel sums, Jacobi variety, and theta function in the scattering case Invent. Math. 99 483-544

Flaschka H, McLaughlin D W and Forest M G 1980 Multiphase averaging and the inverse spectral solution of the Korteweg-de Vries equation Commun. Pure Appl. Math. 33739

Gelfand I M and Dikey L A 1975 Asymptotics of of the resolvent of Sturm-Liouville's equations and algebra of the Korteweg-de Vries equation Usp. Mat. Nauk. 30 67-100

Jin S, Levermore C D and McLaughlin D W 1994 The Behaviour of the Solutions of the NLS Equation in the Semiclassical Limit, Singular Limits of Dispersive Waves (NATO ASI Series B 320) ed N M Ercolani et al (New York: Plenum) pp 21-38

Knörrer H 1982 Geodesics on quadrics and a mechanical problem of C Neumann, J. Reine Angew. Math. 355 69-78

Lax P D and Levermore C D 1983 The small dispersion limit of the Korteweg-de Vries equation I, II, III, Commun. Pure Appl. Math. 36 253-90, 571-93, 809-29.

Lax P D, Levermore C D and Venakidis S 1993 The generation and propagation of oscillations in dispersive IVPs and their limiting behaviour Important Developments in Soliton Theory 1980-1990 ed T Focas and V E Zakharov (New York: Springer) pp 205-41

Moser J 1980 Various aspects of integrable Hamiltonian systems Dynamical Systems (CIME Lectures 1978, Prog. Math. 8) ed J Guckenheimer, J Moser and S Newhouse (Boston, MA: Birkhäuser) pp 233-89

1981 Integrable Hamiltonian systems and spectral theory Lezioni Fermiane Accademia Nazionale dei Lincei, Pisa

Mumford D 1983 Tata Lectures on Theta I and II (Progress in Mathematics 28 and 43) (Boston, MA: Birkhauser)

Newell A C 1985 Solitons in Mathematics and Physics (Regional Conf. Series in Appl. Math. 48) (Philadelphia, PA: SIAM)

Robbins J M 1991 Maslov indices in the Gutzwiller trace formula Nonlinearity 4 343-63 